\title{
La intensidad del esfuerzo en clases de Educación Física Relación entre percepción y cuantificación objetiva de estudiantes y profesores
}

Jaime Carcamo-Oyarzun, Vanessa Carrasco-Alarcon, Jose Miguel Espinoza Silva, Cristian Martinez-Salazar

Universidad de La Frontera (Chile)

Resumen. El presente estudio tiene como objetivos establecer la relación entre percepción subjetiva de esfuerzo con la medición objetiva del esfuerzo a través de acelerometría; así como contrastar la percepción del esfuerzo físico que los escolares tienen de sus clases de Educación Física y la de los profesores que las dirigen. Se monitorearon 7 clases de Educación Física, en donde participaron 56 escolares (21 niños, 35 niñas) de entre 9 y 12 años de edad $(M=11,2 \mathrm{DE}=0,72$ ), y 5 profesores (edad $\mathrm{M}=33,2 \mathrm{DE}=4.26$ ). Se utilizó la «Escala de medición de esfuerzo percibido infantil (EPInfant)» validada para la población escolar chilena; mientras que para la medición objetiva del esfuerzo se consideró el nivel de actividad física a través de acelerometría, medido a través de dispositivos Bioharness 3.0 de la firma Zephyr ${ }^{\mathrm{TM}}$. Los escolares manifestaron una percepción de esfuerzo menor $(\mathrm{M}=3,4 \mathrm{DE}=1,49)$ en relación a sus profesores de Educación Física (M=4,6 DE=0,50), encontrándose diferencias estadísticamente significativas, mientras que el análisis de correlación realizado entre niveles de actividad física y la percepción de esfuerzo arrojó como resultado que existe una correlación estadísticamente significativa pero débil ( $r=0.338, p=0,011)$ entre ambas variables. Estos resultados siguen la tendencia evidenciada en los pocos estudios que han abordado este tema, estimándose que la percepción de esfuerzo tiene potencial como herramienta de evaluación, sin embargo es necesario reafirmar la evidencia de su utilidad en clases de Educación Física.

Palabras clave: percepción del esfuerzo, intensidad, acelerometría, educación física, actividad física.

Abstract. The present study aims to establish the relationship between subjective perception of effort with the objective measurement of effort through accelerometer; as well as to contrast the perception of physical effort that schoolchildren have of their Physical Education classes and that of teachers who teach them. Seven monitored Physical Education classes were monitored, where they participated 56 (21 school children, 35 girls) between 9 and 12 years of age $(M=11.2 D S=0.72$ ), and 5 teachers (age $M=33.2 S D=4.26$ ). We used the «Scale of measurement of perceived exertion (EPInfant)» validated for the Chilean school population; while for the objective measurement of the effort it was considered the level of physical activity through accelerometer, measured through devices Bioharness, Zephyr $^{\mathrm{TM}}$. The students expressed a perception of less effort $(\mathrm{M}=3.4=1.49)$ in relation to their Physical Education Teachers $(\mathrm{M}=4.6=0.50)$ was statistically significant differences, whereas the correlation analysis between levels of physical activity and perception of effort resulted in that there is a weak but statistically significant correlation ( $r=0,338, p=0.011)$ between both variables. These results continue the trend evidenced in the few studies that have addressed this issue, and it is estimated that the perception of effort has potential as an evaluation tool; however it is necessary to reaffirm the evidence of its usefulness in Physical Education classes.

Keywords: perceived exertion, intensity, accelerometry, physical education, physical activity.

\section{Introducción}

La Percepción del Esfuerzo (PE) es una medida psicofisiológica que permite evaluar de manera subjetiva la intensidad de un determinado esfuerzo, tensión o fatiga que se experimenta durante un ejercicio físico realizado (American College of Sports Medicine, 2014). Este concepto, popularizado por Gunnar Borg a fines de 1950 y principios de 1960 (Borg, 1959; Borg, \& Dahlstrom, 1960), es en la actualidad una medida utilizada ampliamente en los ámbitos de la actividad física, deportes y rehabilitación (Pereira, Souza, Reichert, \& Smirmaul, 2014). La PE se ha transformado en materia de estudio tanto en el campo de la fisiología como en el de la psicología, siendo validado como un indicador muy fiable debido a su alta correlación con parámetros fisiológicos de intensidad (Green, Crews, Bosak, \& Peveler, 2003; Scherr, et al., 2013).

La PE es utilizada para la determinación de la intensidad de ejercicio con variados propósitos, como la prescripción de la intensidad del ejercicio (R. Robertson, Goss, \& Metz, 1998; R. Robertson \& Noble, 1997), el control de las cargas de entrenamiento (Murillo, Álvarez, \& Manomelles, 2016;

Fecha recepción: 13-10-18. Fecha de aceptación: 28-04-19 Jaime Carcamo Oyarzun

jaime.carcamo@ufrontera.cl
Sweet, Foster, McGuigan, \& Brice, 2004), e incluso para la predicción de la capacidad de ejercicio máxima (Faulkner, Parfitt, \& Eston, 2007). Si bien es cierto que la PE se ha transformado en una herramienta muy útil para la planificación del entrenamiento de personas sanas y deportistas (Álvarez, Murillo, Usan, Ros, \& Manonelles, 2016; Campos \& Toscano, 2017; Eston, 2012; Guijarro, de la Vega, \& del Valle, 2009; Guillén \& Ruiz-Alfonso, 2015; Pedro \& Martins, 2017) así como para la prescripción clínica en pacientes con distintas enfermedades o patologías (Noble, 1982; Whaley, Brubaker, Kaminsky, \& Miller, 1997), esta evaluación ha demostrado su utilidad en diversas poblaciones, como por ejemplo en adultos mayores (Julius, Brach, Wert, \& VanSwearingen, 2012) o en adultos trabajadores (Habibi, Dehghan, Moghiseh, \& Hasanzadeh, 2014; Hagen \& Harms-Ringdahl, 1994), por lo que no es de extrañar que su utilización también se haya extendido para la evaluación del esfuerzo en la población infantil (Lamb \& Eston, 1997; Robert Robertson et al., 2003; I. Rodríguez \& Gatica, 2016; Utter, Robert Robertson, Nieman, \& Kang, 2002; Williams, Eston, \& Furlong, 1994; Yelling, Lamb, \& Swaine, 2002).

Ya en la década de los 70, Bar-Or (1977) manifestaba que la PE en niños seguía el mismo comportamiento que la PE en adultos, es decir, ascendía de forma lineal mientras se incrementaba la intensidad del ejercicio; sin embargo, los 
niños tenderían a reportar una baja PE en una determinada intensidad de ejercicio comparado con los adultos (Lamb, Parfitt, \& Eston, 2013). La evaluación de la PE en niños a través de las escalas utilizadas en adultos no sería tan efectiva, debido a la alta demanda de comprensión de las expresiones verbales y su relación con la categoría numérica correspondiente a las exigencias fisiológicas, por lo que se hizo necesario adaptar las escalas para la población infantil (Williams, et al., 1994). En un principio esta adaptación se realizó a través de formas numéricas y expresiones verbales mucho más simples, obteniendo asociaciones positivas entre la PE y la frecuencia cardiaca en ejercicios progresivos (Williams, et al., 1994). Posteriormente se han desarrollado diversas escalas que incluyen pictogramas que facilitar la identificación del nivel de esfuerzo realizado en ejercicios, mostrando altos niveles de validez y fiabilidad (Marinov, Mandadjieva, \& Kostianev, 2008; Rodríguez, Zambrano, \& Manterola, 2016; Roemmich et al., 2006; Yelling et al., 2002). Así, se establece que las escalas para la población infantil que representan los diferentes niveles de intensidad a través de ilustraciones gráficas, arrojan una mayor validez de criterio concurrente en comparación a las escalas numéricas para adultos (Rodríguez et al., 2016). En ese contexto, la PE en niños y niñas también se ha utilizado para determinar la intensidad del esfuerzo en el campo de la pediatría, como prescripción del ejercicio aeróbico (Lambrick, Rowlands, \& Eston, 2011; Verweij, Stoner, \& Shultz, 2013), ejercicios de fuerza (Peña, Heredia, Lloret, Martín, \& Silva-Grigoletto, 2016) y resistencia (Faigenbaum, Milliken, Cloutier, \&Westcott, 2004). Asimismo, ha sido utilizado como herramienta para la descripción del esfuerzo en actividades deportivas infantiles (Fuentes Azpiroz, Feu, Jiménez, \& Calleja-González, 2013; Morales Belando \& Arias-Estero, 2015). Considerando esta diversidad de ámbitos de uso, la PE también se ha utilizado como una herramienta para el control de la intensidad en clases de Educación Física, aunque aún no ha sido muy abordada desde el ámbito científico, a pesar de ser una herramienta didáctica y de estar recomendado en los programas de estudio (Ministerio de Educación de Chile, 2012, p.60). La evidencia científica sobre su uso en clases de Educación Física no es muy amplia (Hernández-Álvarez, del-CampoVecino, Martínez-de-Haro, \& Moya-Morales. J.M., 2010), en donde los escasos estudios la han relacionado con la variables objetiva de frecuencia cardiaca (Hernández-Álvarez et al., 2010; Lagally, 2013; Lagally, Walker-Smith, Henninger, Williams, \& Coleman, 2016). Incluso en algunos currículos de enseñanza de la Educación Física, como en las bases curriculares de Chile, se recomienda su uso (Ministerio de Educación de Chile, 2012, p.182), debido al enfoque que actualmente ha adoptado esta asignatura, en donde los objetivos ya no solo tratan de desarrollar las habilidades motrices y promover los hábitos de vida activos entre los escolares sino que también hacen énfasis en potenciar los efectos que la actividad física puede producir en el organismo (CarcamoOyarzun, Wydra, Hernandez-Mosqueira, \& Martinez-Salazar, 2017), proponiendo a través de orientaciones didácticas que el desarrollo de las clases gire en torno a la práctica de actividades que se ejecuten con una intensidad de moderada a vigorosa la mayor cantidad de tiempo posible (Ministerio de Educación de Chile, 2013, p.115). Desde este punto de vista, el control de la intensidad de la actividad física cobra relevancia dentro de las clases de Educación Física, lo que podría realizarse a través de métodos objetivos, con instrumentos como monitores de frecuencia cardiaca o acelerómetros, sin embargo, estos métodos presentan diversas dificultades para su uso en clases, como su alto costo y la gran cantidad de tiempo para su preparación y retiro (Lagally, 2013; Lagally, \& Walker-Smith, 2010). Es aquí en donde la PE se perfila como una útil herramienta para la medición de la intensidad, ya que no presenta costos, no necesita de tiempo para su instalación y el rango de medición es el mismo para todos los estudiantes (Lagally, 2013). Además de estas ventajas, es necesario considerar que es posible evaluar simultáneamente a grandes grupos de escolares (Hernández-Álvarez, et al., 2010), por lo que el uso de la escala de PE permite al profesor recibir una retroalimentación de los efectos de su clases, y también los escolares puedan utilizar estas escalas como control propio de la intensidad cuando realicen actividad física (Lagally, 2013), uno de los objetivos de aprendizaje que se plantean dentro del currículo de Educación (Ministerio de Educación de Chile, 2012, p.182). En ese marco, el presente estudio tiene por propósito, por un lado, relacionar la percepción subjetiva de esfuerzo de los escolares con la medición objetiva del esfuerzo a través de acelerometría; y por otro lado, se pretende contrastar la PE que los escolares reportan, versus la percepción de los profesores de Educación Física respecto al esfuerzo que los escolares realizan en sus clases.

\section{Material y Método}

\section{Muestra}

Participaron escolares de cuarto, quinto y sexto año de Enseñanza Básica de cuatro establecimientos educacionales de la ciudad de Coyhaique, Región de Aysén, Chile. Se monitorearon siete clases de Educación Física. Como criterio de inclusión se consideró a todos los escolares que participaran de las clases de Educación Física y que no hayan presentado certificado médico. Se seleccionaron aleatoriamente por sorteo a 60 escolares para la medición del esfuerzo objetivo, de los cuales se excluyeron a 4 por no participar de la totalidad de la sesión. En total, la muestra consideró a 56 escolares (21 niños, 35 niñas) de entre nueve y 12 años de edad $(\mathrm{M}=11,2 \mathrm{DE}=0,72)$. En relación a los profesores, participaron los cinco docentes que dirigieron las siete clases (dos profesores realizaron dos clases), todos profesores de Educación Física (edad M = 33,2 DE=4.26).

\section{Instrumentos}

Para la medición de la PE realizado en la clase de Educación Física se utilizó la «Escala de medición de esfuerzo percibido infantil (EPInfant)» validada para la población escolar chilena (Rodríguez et al., 2015; Rodríguez, 2016; Rodríguez \& Gatica, 2016), la que presenta altos índices de validez de contenido, de criterio concurrente y confiabilidad en los diversos análisis estadísticos aplicados (Rodríguez-Núñez, \& Manterola, 2016). Esta escala ofrece la opción de elegir un valor representativo del esfuerzo físico realizado, dentro de una escala de descriptores numéricos de 1 a 10, apoyados por descriptores verbales e ilustraciones que representan a 
un niño corriendo a intensidades crecientes a lo largo de una escala de barras.

Para la medición objetiva del esfuerzo se consideró el nivel de Actividad Física a través de acelerometría, medido a través de dispositivos Bioharness 3.0 PSM (Physiological Status Monitoring) de la firma Zephyr ${ }^{\mathrm{TM}}$. Este sistema ha sido validado por diversos estudios tanto en laboratorio (Brooks, Carter, \& Dawes, 2013; Hailstone, \& Kilding, 2011; Johnstone, Ford, Hughes, Watson, \& Garrett, 2012a, 2012b) como en campo (Johnstone et al., 2012). El sistema Bioharness 3.0 consta de un módulo compacto de monitoreo fisiológico instalado en un cinturón, situado a la altura de la apófisis xifoides del esternón del sujeto evaluado y en contacto con la piel, de donde envía información a una interface conectada al Software Omnisense ${ }^{\mathrm{TM}}$ que permite ver la información del evaluado en tiempo real, además de grabar todos los datos en el mismo biomódulo, para su posterior análisis. El Nivel de Actividad Física se determina a través de Unidades de Magnitud Vectorial (VMU), en donde la aceleración se expresa en fuerza gravitacional (g), la que es calculada automáticamente en el Software Omnisense a través de la información que el Bioharness 3.0 envía por acelerometría en tres ejes (1Hz) (Zephyr Technology, 2012). Los diferentes niveles se clasifican de la siguiente forma: Actividad Baja = VMU entre 0 y $0.2 \mathrm{~g}$, equivalente a estar de pie, sin moverse; Actividad Leve $=$ VMU entre 0.2 y $0.8 g$, equivalente a caminar; y Actividad Elevada= VMU mayor a 0.8g, equivalente a correr.

\section{Procedimiento}

Se contactó a los Directores de los Establecimientos Educacionales para solicitar una reunión en donde se presentaría el estudio. Posteriormente se procedió a tomar contacto con los profesores de Educación Física, para informar sobre el estudio y solicitar su colaboración.

Teniendo en cuenta que los monitoreos se realizarían en sesiones de 90 minutos, se acordó con los profesores dejar 30 minutos de organización (llegada de los escolares al gimnasio, control de asistencia, orden y distribución de material, explicación de los objetivos de la clase, instalación de los cinturones a los alumnos seleccionados, etc.), y se les solicitó que planificaran una clase de 60 minutos, que sería el tiempo considerado para la medición. Los monitoreos se realizaron en clases regulares de educación física correspondiente al periodo de la Unidad Didáctica de Habilidades Motrices.

Previo al desarrollo del estudio, se realizaron dos visitas previas a los escolares. En la primera se les presento el estudio, y se les entregó el asentimiento informado. En la segunda visita se realizó la presentación e inducción de la escala EPInfant y como se determinada la intensidad, familiarizándose con ella a través de ejercicios prácticos.

Se les explicó a los escolares que, considerando que solo se contaban con 10 equipos Bioharness, se elegirían vía sorteo a los 10 estudiantes que utilizarían los equipos durante la clase. Con los estudiantes sorteados se realizó un ensayo para la instalación de los cinturones, en donde las niñas fueron instruidas por una profesora, mientras que los niños por un profesor.

En las sesiones de monitoreo, se instalaron los equipos en los escolares previamente sorteados, quienes se unieron al resto de su grupo y participaron con normalidad de la clase de 60 minutos dirigida por su profesor.

Los datos de intensidad fueron recepcionados en un computador a través del Software Omnisense y guardados para su posterior revisión.

La escala EPInfant fue aplicada tanto a los escolares que participaron de la clase, como al profesor que la dirigió, siguiendo las recomendaciones de Rodríguez y Gatica (2016). Se les entregó una hoja con la escala, con un encabezado que correspondiente a la pregunta: ¿Cuán cansado te encontraste durante la clase? La escala se aplicó una vez finalizada la clase, con el propósito de evitar el efecto olvido.

\section{Consideraciones Éticas}

Para la realización de este proyecto se solicitó la autorización de la Secretaría Regional Ministerial de Educación de la Región de Aysén. Luego de oficializada dicha autorización se presentó a los Directores de los Establecimientos Educacionales y sus respectivos Profesores de Educación Física un documento con el objetivo de la investigación y los procedimientos correspondientes. Se les solicitó un consentimiento informado a los Padres y un asentimiento informado a los escolares que participaron del estudio. El protocolo de investigación fue evaluado y aprobado por el Comité Ético Científico de la Universidad de La Frontera según Acta de Aprobación No15-2017.

\section{Análisis estadístico}

El análisis de los datos fue realizado con el programa estadístico SPSS Statistics, versión 17. La normalidad se evaluó a través de la prueba Kolmogórov-Smirnov. Se realizaron los cálculos estadísticos descriptivos de tendencia central (media) y dispersión (desviación típica). Para determinar la relación entre los niveles de actividad física y esfuerzo percibido se aplicó el coeficiente de correlación de Pearson, mientras que para determinar las diferencias de esfuerzo percibido entre escolares y profesores se utilizó la prueba $t$ de Student. El nivel de significancia fue establecido en .05.

\section{Resultados}

En la Tabla 1 se presentan los resultados correspondientes a la PE indicada por medio de la escala EPInfant, tanto de los escolares como de los profesores. Al considerar el total de casos, los escolares manifestaron una percepción de esfuerzo menor $(\mathrm{M}=3,4 \mathrm{DE}=1,49)$ en relación a sus profesores de Educación Física ( $M=4,6 \mathrm{DE}=0,50)$, encontrándose diferencias estadísticamente significativas $(t(61)=-2.240$, $\mathrm{p}=0.029)$. Al realizar el análisis de concordancia se obtuvo una asociación débil en términos de correlación intra clase (ICC $=0.215$; $-3.40 \cdot 0.540)$.

Los resultados correspondientes a los valores medios de los niveles de actividad realizados por los escolares en cada una de las clases de Educación Física monitoreada se presentan en la Tabla 2. Según la clasificación la categorización de los VMU, el nivel de actividad realizada por los escolares corresponde a «Leve» (VMU entre 0.2 y 0.8g), obteniéndose una media de 0.32 (DE=0.069) considerando al total de los escolares. 
El análisis de correlación realizado entre niveles de actividad física y la percepción subjetiva de esfuerzo se presenta en la Tabla 3, en donde los resultados arrojaron una correlación estadísticamente significativa pero débil entre ambas variables.

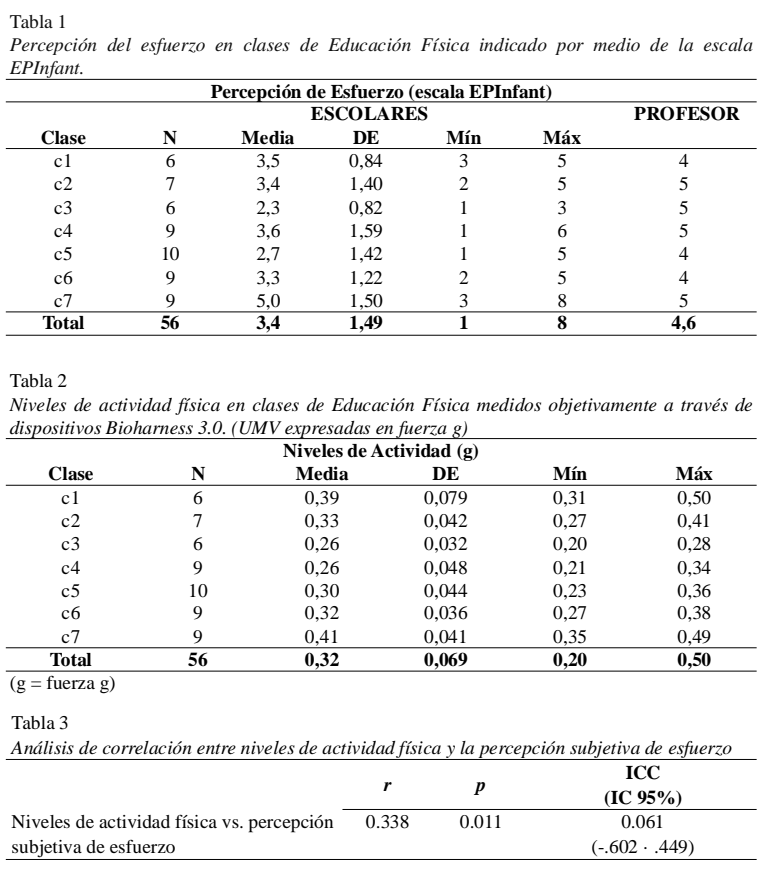

\section{Discusión}

El presente estudio tiene como objetivos establecer la relación entre percepción subjetiva de esfuerzo con la medición objetiva del esfuerzo a través de acelerometría; así como contrastar la PE físico que los escolares tienen de sus clases de Educación Física y la de los profesores que las dirigen.

Los resultados indican que existe una correlación positiva aunque débil entre percepción subjetiva y la medición objetiva del esfuerzo a través de acelerometría. Esto coincide con los resultados obtenidos por Lagally et al. (2016), quienes también buscaban relacionar el esfuerzo subjetivo con el objetivo, pero a diferencia de nuestro estudio, utilizaron la escala pictográfica de percepción de esfuerzo OMNI y la frecuencia cardíaca medida con cardiofrecuenciometros. Estos autores analizaron clases de Educación Física en donde se realizaron circuitos con ejercicios cardiovasculares y de resistencia muscular monitoreando a escolares de entre 11 y 13 años, encontrándose correlaciones significativas pero débiles entre las medias de la escala de percepción de esfuerzo y la frecuencia cardíaca, independiente del tipo de circuito realizado (cardiovascular $r=.33, p=.02$; muscular $r=.38, p=01$ ). Otros estudios como los de Robertson et al. (2005) y Gillach, Sallis, Buono, Patterson, y Nader (1989), que también relacionaron el esfuerzo percibido y la frecuencia cardiaca en niños de entre 10 y 14 años, arrojaron correlaciones más fuertes entre ambas variables (entre $r=.65 \mathrm{y} r=89$ ), pero estos estudios fueron realizados de manera controlada en laboratorios, en donde las intensidades se incrementaban a medida que pasaba el tiempo. No obstante, tanto nuestro estudio como el de Lagally et al. (2016) considera un diseño que buscaba abordar clases de Educación Física lo más cercanas a la realidad, por lo que la intensidad no podría ser controlada o incrementada de forma graduada, lo que explicaría que las correlaciones sean menores que las de estudios realizados en laboratorios; sin embargo, los resultados de ambos estudios coinciden con la tendencia que relaciona los parámetros de esfuerzo objetivos con los subjetivos.

En referencia al segundo objetivo de este estudio, los resultados indican existen diferencias significativas en la PE físico que los escolares tienen de sus clases de Educación Física y la de los profesores que las dirigen, en donde los profesores de Educación Física perciben que en sus clases se realiza un esfuerzo físico mayor $(M=4,6)$, en relación a la percepción de esfuerzo que los propios escolares reportan $(\mathrm{M}=3,4)$. Estos resultados coinciden con los obtenidos por Wydra (2009, 2010), quien combino observación de clases, medición de la frecuencia cardíaca y percepción de la intensidad de la clases tanto por parte de los escolares como por la de los docentes. Wydra (2009) observo 237 cursos de $3^{\circ}$ de primaria hasta $13^{\circ}$ de secundaria ( $M=13,7$ años), lo que equivalió a un total de 4.524 escolares, utilizándose la escala de Borg de seis a 20 para determinación de la percepción de esfuerzo. La calificación de los alumnos alcanzo una media de 11,3 (poco intenso), mientras que la percepción de los profesores fue de 13,5 (algo intenso) determinándose diferencias significativas. En un estudio posterior, realizado con 443 alumnos de 46 cursos de $5^{\circ}$ a $12^{\circ}$ año, Wydra (2010) encontró resultados similares al evaluar la percepción de esfuerzo con escala de Borg de seis a 20, en donde la percepción de los escolares fue de 11,1 (poco intensa) y la de los profesores en 13,0 (algo intensa). Estos resultados sugieren que los profesores, al planificar sus clases, podrían estar sobreestimando la intensidad de las actividades a realizar, lo que restaría eficacia a las practicas pedagógicas que buscan reconocer las respuestas corporales asociadas a la actividad física (Wydra, 2010).

A pesar de los interesantes hallazgos, es importante indicar las diversas limitaciones de este estudio, como por ejemplo el número de la muestra, que, según Lagally et al. (2016) un bajo número de participantes limita el análisis de correlación. Asimismo, los datos corresponden al monitoreo de la participación de escolares en una sola clase, a lo que Lagally et al. (2016) plantea que al analizar una mayor cantidad de clases se podrían obtener resultados más concluyentes.

La recopilación de datos en una clase regular de Educación Física también impide el control adecuado de factores importantes como pueden ser la intensidad y el volumen de las cargas, a diferencia de las evaluaciones en laboratorio en donde se demuestra una alta relación entre acelerometría y esfuerzo percibido, sin embargo los beneficios potenciales de la utilización de escalas de esfuerzo percibido en las clases de Educación Física son numerosas tanto para los escolares como para los profesores. En comparación con los acelerómetros, monitores de frecuencia cardíaca u otras tecnologías, las mediciones subjetivas de percepción de esfuerzo pueden aumentar la motivación, ya que proporcionan a los escolares un sentido de control y autonomía sobre su rendimiento en el ejercicio (Lagally et al., 2016), lo que se ha vinculado a la mejora y disfrute, fomentando la adherencia a la actividad física (Partridge, King, \& Bian, 2011).

Los resultados de este estudio también proporcionan a 
los profesores un método para abordar los objetivos planteados en el curriculum de Educación Física, especialmente a los relacionados con las respuestas corporales asociadas a la actividad física (Ministerio de Educación de Chile, 2013, p.186). En el caso de Chile, las Bases Curriculares de Educación Física y Salud plantean un objetivo específico relacionado con la percepción de esfuerzo (Objetivo de Aprendizaje $\left.N^{\circ} 8\right)$ : «Determinar la intensidad del esfuerzo físico de forma manual, mediante el pulso o utilizando escalas de percepción de esfuerzo» (Ministerio de Educación de Chile, 2013, p.186), por lo que las escalas de percepción de esfuerzo se presentarían como una herramienta para lograr este objetivo curricular.

\section{Conclusiones}

Existe una correlación significativa baja entre la percepción subjetiva y la medición objetiva del esfuerzo a través de acelerometría, siguiendo la tendencia evidenciada en los pocos estudios que han abordado este tema. Por otro lado, los profesores de Educación Física perciben que en sus clases se realiza un esfuerzo físico mayor, en relación a la percepción de esfuerzo que los propios escolares reportan. En necesario profundizar más en esta temática para poder establecer la relación entre parámetros subjetivos y objetivos de esfuerzo físico en clases de Educación Física, considerando diversas tecnologías como cardiofrecuenciometros y acelerómetros; así como el monitoreo de varias clases, que traten diferentes unidades didácticas, que sean dirigidas por diversos profesores y en distintos contextos, para poder establecer un panorama más amplio y tangible del uso de las escalas subjetivas. La percepción de esfuerzo tiene potencial como herramienta de evaluación, sin embargo, es necesario reafirmar la evidencia de su utilidad en clases de Educación Física.

\section{Agradecimientos}

A la Corporación Municipal de Deportes de Coyhaique; al Plan de desarrollo de la motricidad como potenciador de los aprendizajes y estado nutricional de la Unidad de Gestión Pedagógica de la Municipalidad de Temuco; y al Centro de Evaluación Física del Centro de Entrenamiento Regional IND de Aysén.

\section{Financiamiento}

Proyecto Financiado por la Dirección de Investigación de la Universidad de La Frontera, a través del Proyecto DIUFRO Código DI17-0104 titulado «Cuantificación y Percepción del esfuerzo físico realizado por escolares en clases de Educación Física».

\section{Referencias}

Álvarez, J., Murillo, V., Usan, P., Ros, R., \& Manonelles, P. (2016). Percepción subjetiva como método de control de la intensidad en fútbol sala. RETOS. Nuevas Tendencias En Educación Física, Deporte Y Recreación. (30), 9-14. Retrieved from https://recyt.fecyt.es/index.php/retos/ article/download/35128/30084

American College of Sports Medicine. (2014). Guidelines for exercise testing and prescription (9th ed.). Philadelphia: Wolters Kluwer/Lippincott Williams \& Wilkins Health.

Bar-Or, O. (1977). Age-related changes in exercise perception. Physical Work and Effort G. Borg (ED), 255-256.

Borg, G. (1959). Psykofysisk undersökning av arbete pa cykelergometer. Nordisk Medicin, 62, 1383-1386.

Borg, G, \& Dahlstrom, H. (1960). The perception of muscular work. Umea Veten Skapliga Skrifserie, 5, 1-26.

Brooks, K.A., Carter, J. G, \& Dawes, J. J. (2013). AComparison of VO2 Measurement Obtained by a Physiological Monitoring Device and the Cosmed Quark CPET. Journal of Novel Physiotherapies, 3(1), 1-2. https://doi.org/ 10.4172/2165-7025.1000126

Campos, M. Á., \& Toscano, F. J. (2017). Comparación de la percepción subjetiva del esfuerzo entre partidos amistosos y diferentes tipos de sesión en futbolistas profesionales. RETOS. Nuevas Tendencias En Educación Física, Deporte Y Recreación. (34), 66-70. Retrieved from https://recyt.fecyt.es/index.php/retos/article/download/ $55248 / 36822$

Carcamo-Oyarzun, J., Wydra, G., Hernandez-Mosqueira, C., \& Martinez-Salazar, C. M. (2017). Actitudes hacia la educación física: Grados de importancia y conformidad según escolares de Chile y Alemania. Una mirada intercultural. RETOS: Nuevas Tendencias En Educación Física, Deporte Y Recreación(32), 158-162. Retrieved from https://recyt.fecyt.es/index.php/retos/article/view/ 52824

Eston, R. (2012). Use of ratings of perceived exertion in sports. International Journal of Sports Physiology and Performance, 7(2), 175-182.

Faigenbaum, A. D., Milliken, L. A., Cloutier, G., \& Westcott, W. L. (2004). Perceived exertion during resistance exercise by children. Perceptual and Motor Skills, 98(2), 627637. https://doi.org/10.2466/pms.98.2.627-637

Faulkner, J., Parfitt, G., \& Eston, Roger. (2007). Prediction of maximal oxygen uptake from the ratings of perceived exertion and heart rate during a perceptually-regulated sub-maximal exercise test in active and sedentary participants. European Journal of Applied Physiology, 101(3), 397-407.https://doi.org/10.1007/s00421-007-05086

Fuentes Azpiroz, M., Feu, S., Jiménez, C., \& Calleja-González, J. (2013). Perceived exertion effort in mini basketball players and its relationship with training volume. Revista De Psicología Del Deporte, 22(1). Retrieved from http://www.redalyc.org/pdf/2351/235127552022.pdf

Gillach, M. C., Sallis, J. F., Buono, M. J., Patterson, P., \& Nader, P. R. (1989). The Relationship between Perceived Exertion and Heart Rate in Children and Adults. Pediatric Exercise Science, 1(4), 360-368. https://doi.org/10.1123/ pes.1.4.360

Green, J. M., Crews, T. R., Bosak, A. M., \& Peveler, W. W. (2003). Overall and differentiated ratings of perceived exertion at the respiratory compensation threshold: Effects of gender and mode. European Journal of Applied Physiology, 89(5), 445-450. https://doi.org/ 10.1007/s00421-003-0869-4 
Guijarro, E., La Vega, R. de, \& del Valle, S. (2009). Ciclo menstrual, rendimiento y percepción del esfuerzo en jugadoras de fútbol de élite. Revista Internacional De Medicina Y Ciencias De La Actividad Física Y El Deporte, 9(34), 94-104.

Guillén, F., \& Ruiz-Alfonso, Z. (2015). Influencia de la música en el rendimiento físico, esfuerzo percibido y motivación / Influence of Music on Physical Performance, Perceived Exertion and Motivation. Revista Internacional De Medicina Y Ciencias De La Actividad Física Y Del Deporte, 60, 701-717. https://doi.org/10.15366/ rimcafd2015.60.006

Habibi, E., Dehghan, H., Moghiseh, M., \& Hasanzadeh, A. (2014). Study of the relationship between the aerobic capacity (VO2 max) and the rating of perceived exertion based on the measurement of heart beat in the metal industries. Journal of Education and Health Promotion, 3, 55. https://doi.org/10.4103/2277-9531.134751

Hagen, K. B., \& Harms-Ringdahl, K. (1994). Ratings of Perceived Thigh and Back Exertion in Forest Workers During Repetitive Lifting Using Squat and Stoop Techniques. Spine, 19(22). Retrieved from https:// journals.lww.com/spinejournal/Fulltext/1994/11001/ Ratings_of_Perceived_Thigh_and_Back_Exertion_in.4.aspx

Hailstone, J., \& Kilding, A. E. (2011). Reliability and Validity of the Zephyr ${ }^{\mathrm{TM}}$ BioHarness ${ }^{\mathrm{TM}}$ to Measure Respiratory Responses to Exercise. Measurement in Physical Education and Exercise Science, 15(4), 293-300. https:/ /doi.org/10.1080/1091367X.2011.615671

Hernández-Álvarez, J. L., del-Campo-Vecino, J., Martínez-deHaro, V., \& Moya-Morales. J.M. (2010). Percepción de esfuerzo en Educación Física y su relación con las directrices sobre actividad física. Revista Internacional De Medicina Y Ciencias De La Actividad Física Y El Deporte, 10(40), 609-619.

Johnstone, J. A., Ford, P. A., Hughes, G. Watson, T., \& Garrett, A. T. (2012a). Bioharness( ${ }^{\mathrm{TM}}$ ) Multivariable Monitoring Device: Part. II: Reliability. Journal of Sports Science \& Medicine, 11(3), 409-417.

Johnstone, J. A., Ford, P. A., Hughes, G. Watson, T., \& Garrett, A. T. (2012b). Bioharness ${ }^{\text {TM }}$ Multivariable Monitoring Device: Part. I: Validity. Journal of Sports Science \& Medicine, 11(3), 400-408.

Johnstone, J.A., Ford, P. A., Hughes, G., Watson, T., Mitchell, A. C. S., \& Garrett, A. T. (2012). Field based reliability and validity of the bioharness ${ }^{\mathrm{TM}}$ multivariable monitoring device. Journal of Sports Science \& Medicine, 11(4), 643-652.

Julius, L. M., Brach, J. S., Wert, D. M., \& VanSwearingen, J. M. (2012). Perceived effort of walking: Relationship with gait, physical function and activity, fear of falling, and confidence in walking in older adults with mobility limitations. Physical Therapy, 92(10), 1268-1277. https:/ /doi.org/10.2522/ptj.20110326

Lagally, K. M. (2013). Using Ratings of Perceived Exertion in Physical Education. Journal of Physical Education, Recreation \& Dance, 84(5), 35-39. https://doi.org/ 10.1080/07303084.2013.779533

Lagally, K. M., \& Walker-Smith, K. (2010). The Validity Of Using Ratings Of Perceived Exertion To Monitor Intensity
During Physical Education Classes. Medicine and Science in Sports and Exercise, 42, 670. https://doi.org/ 10.1249/01.MSS.0000385868.82761.7b

Lagally, K. M., Walker-Smith, K., Henninger, M. L., Williams, S., \& Coleman, M. (2016). Acute and Session Ratings of Perceived Exertion in a Physical Education Setting. Perceptual and Motor Skills, 122(1), 76-87. https:// doi.org/10.1177/0031512515625376

Lamb, K. L., \& Eston, R. G. (1997). Effort Perception in Children. Sports Medicine, 23(3), 139-148. https://doi.org/10.2165/ 00007256-199723030-00001

Lamb, K. L., Parfitt, G., \& Eston, R. (2013). Effort perception. In N. Armstrong \& W. van Mechelen (Eds.), Paediatric exercise science and medicine (2nd ed., pp. 145-154). [Oxford], [Oxford]: Oxford University Press.

Lambrick, D. M., Rowlands, A. V., \& Eston, R. G (2011). The Perceptual Response to Treadmill Exercise Using the Eston-Parfitt Scale and Marble Dropping Task, in Children Age 7 to 8 Years. Pediatric Exercise Science, 23(1), 3648. https://doi.org/10.1123/pes.23.1.36

Marinov, B., Mandadjieva, S., \& Kostianev, S. (2008). Pictorial and verbal category-ratio scales for effort estimation in children. Child: Care, Health and Development, 34(1), 35-43. https://doi.org/10.1111/j.1365-2214.2007.00767.x

Morales Belando, M., \& Arias-Estero, J. L. (2015). Diferencias entre el juego 7 vs. 7 y el 4 vs. 4 en el balonmano escolar en relación al rendimiento, percepción del esfuerzo y la intencionalidad de práctica. RETOS. Nuevas Tendencias En Educación Física, Deporte Y Recreación. (27). Retrieved from http://www.redalyc.org/pdf/3457/ 345738764007.pdf

Murillo, V., Álvarez, J., \& Manomelles, P. (2016). Control of training loads through perceived exertion. Prediction of heart rate. Retos. (30), 82-86.

Noble, B. J. (1982). Clinical applications of perceived exertion. Medicine and Science in Sports and Exercise, 14(5), 406-411.

Partridge, J. A., King, K. M., \& Bian, W. (2011). Perceptions of Heart Rate Monitor Use in High School Physical Education Classes. Physical Educator, 68(1), 30-43.

Pedro, S., \& Martins, P. (2017). Suporte de autonomia, empenhamento e perceção subjetiva de esforço em lutadores. Revista Iberoamericana De Psicología Del Ejercicio Y El Deporte, 12(2), 279-286.

Peña, G., Heredia, J. R., Lloret, C., Martín, M., \& SilvaGrigoletto, M. D. (2016). Iniciación al entrenamiento de fuerza en edades tempranas: revisión. Revista Andaluza De Medicina Del Deporte, 9(1), 41-49. https://doi.org/ 10.1016/j.ramd.2015.01.022

Pereira, G., Souza, D. M. d., Reichert, F. F., \& Smirmaul, B. P. C. (2014). Evolução dos conceitos e mecanismos da percepção de esforço: Uma revisão de literatura. Revista Brasileira De Cineantropometria E Desempenho Humano, 16(5), 579. https://doi.org/10.5007/19800037.2014v16n5p579

Robertson, R., Goss, F., \& Metz, K. (1998). Perception of physical exertion during dynamic exercise: A tribute to Professor Gunnar A. V. Borg. Perceptual and Motor Skills, 86(1), 183-191. https://doi.org/10.2466/ pms.1998.86.1.183 
Robertson, R., \& Noble, B. J. (1997). Perception of physical exertion: Methods, mediators, and applications. Exercise and Sport Sciences Reviews, 25, 407-452.

Robertson, R., Goss, F., Andreacci, J. L., Dubé, J. J., Rutkowski, J., Frazee, K. M., . . . Snee, B. M. (2005). Validation of the Children's OMNI-Resistance Exercise Scale of perceived exertion. Medicine and Science in Sports and Exercise, 37(5), 819-826.

Robertson, R., Goss, F., Rutkowski, J., Lenz, B., Dixon, C., Timmer, J., ... Andreacci, J. (2003). Concurrent validation of the OMNI perceived exertion scale for resistance exercise. Medicine and Science in Sports and Exercise, 35(2), 333-341. https://doi.org/10.1249/ 01.MSS.0000048831.15016.2A

Rodríguez, I., \& Gatica, D. (2016). Percepción de esfuerzo durante el ejercicio: ¿Es válida su medición en la población infantil? Revista Chilena De Enfermedades Respiratorias, 32(1), 25-33. https://doi.org/10.4067/S071773482016000100005

Rodríguez, I., Zambrano, L., \& Manterola, C. (2016). Criterionrelated validity of perceived exertion scales in healthy children: A systematic review and meta-analysis.

Rodríguez, I. (2016). Escala de medición de esfuerzo percibido infantil (EPInfant): Validación en niños y adolescentes chilenos [Measurement scale of child perceived exertion (EPInfant): Validation Chilean children and adolescents]. Revista Chilena De Pediatria, 87(3), 211212. https://doi.org/10.1016/j.rchipe.2015.09.001

Rodríguez, I., Zenteno, D., Cisternas, L., Rodríguez, P., Reyes, G., \& Troncoso, K. (2015). Construcción y evaluación de Epinfant: Una escala para la medición del esfuerzo percibido en la población pediátrica. Archivos Argentinos De Pediatria, 113(6), 550-557. https://doi.org/ 10.5546/aap.2015.550

Rodríguez-Núñez, I., \& Manterola, C. (2016). Initial validation of the scale of perceived exertion (EPInfant) in Chilean children. Biomedica : Revista Del Instituto Nacional De Salud, 36(1), 29-38. https://doi.org/10.7705/ biomedica.v36i1.2720

Roemmich, J. N., Barkley, J. E., Epstein, L. H., Lobarinas, C. L., White, T. M., \& Foster, J. (2006). Validity of PCERT and OMNI walk/run ratings of perceived exertion. Medicine and Science in Sports and Exercise, 38(5), 1014-1019. https://doi.org/10.1249/01.mss.0000218123.81079.49

Scherr, J., Wolfarth, B., Christle, J. W., Pressler, A., Wagenpfeil,
S., \& Halle, M. (2013). Associations between Borg's rating of perceived exertion and physiological measures of exercise intensity. European Journal of Applied Physiology, 113(1), 147-155. https://doi.org/10.1007/ s00421-012-2421-X

Sweet, T. W., Foster, C., McGuigan, M. R., \& Brice, G. (2004). Quantitation of resistance training using the session rating of perceived exertion method. Journal of Strength and Conditioning Research, 18(4), 796-802. https:// doi.org/10.1519/14153.1

Utter, A. C., Robertson, R., Nieman, D. C., \& Kang, J. (2002). Children's OMNI Scale of Perceived Exertion: Walking/ running evaluation. Medicine and Science in Sports and Exercise, 34(1), 139-144.

Verweij, B. G. F., Stoner, L., \& Shultz, S. P. (2013). Exercise modality and metabolic efficiency in children. European Journal of Pediatrics, 172(9), 1191-1196. https://doi.org/ 10.1007/s00431-013-2025-4

Whaley, M. H., Brubaker, P. H., Kaminsky, L. A., \& Miller, C. R. (1997). Validity of Rating of Perceived Exertion During Graded Exercise Testing in Apparently Healthy Adults and Cardiac Patients. Journal of Cardiopulmonary Rehabilitation and Prevention, 17(4), 261-267. Retrieved from https://journals.lww.com/jcrjournal/

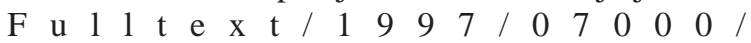
Validity_of_Rating_of_Perceived_Exertion_During.7.aspx Williams, J., Eston, R., \& Furlong, B. (1994). Cert:A perceived exertion scale for young children. Perceptual and Motor Skills, 79(3 Pt 2), 1451-1458. https://doi.org/10.2466/ pms.1994.79.3f.1451

Wydra, G. (2009). Belastungszeiten und Anstrengung im Sportunterricht. Sportunterricht, 58(5), 129-136.

Wydra, G. (2010). Untersuchungen zur Belastungsintensität im Sportunterricht. In P. Frei \& S. Körner (Ed.), Ungewissheit. Sportpädagogische Felder im Wandel (pp. 227-234). Hamburg: Feldhaus.

Yelling, M., Lamb, K. L., \& Swaine, I. L. (2002). Validity of a Pictorial Perceived Exertion Scale for Effort Estimation and Effort Production During Stepping Exercise in Adolescent Children. European Physical Education Review, 8(2), 157-175. https://doi.org/10.1177/ 1356336X020082007

Zephyr Technology. (2012). Bioharness 3.0: User Manual. Retrieved from https://www.zephyranywhere.com/media/ download/bioharness3-user-manual.pdf
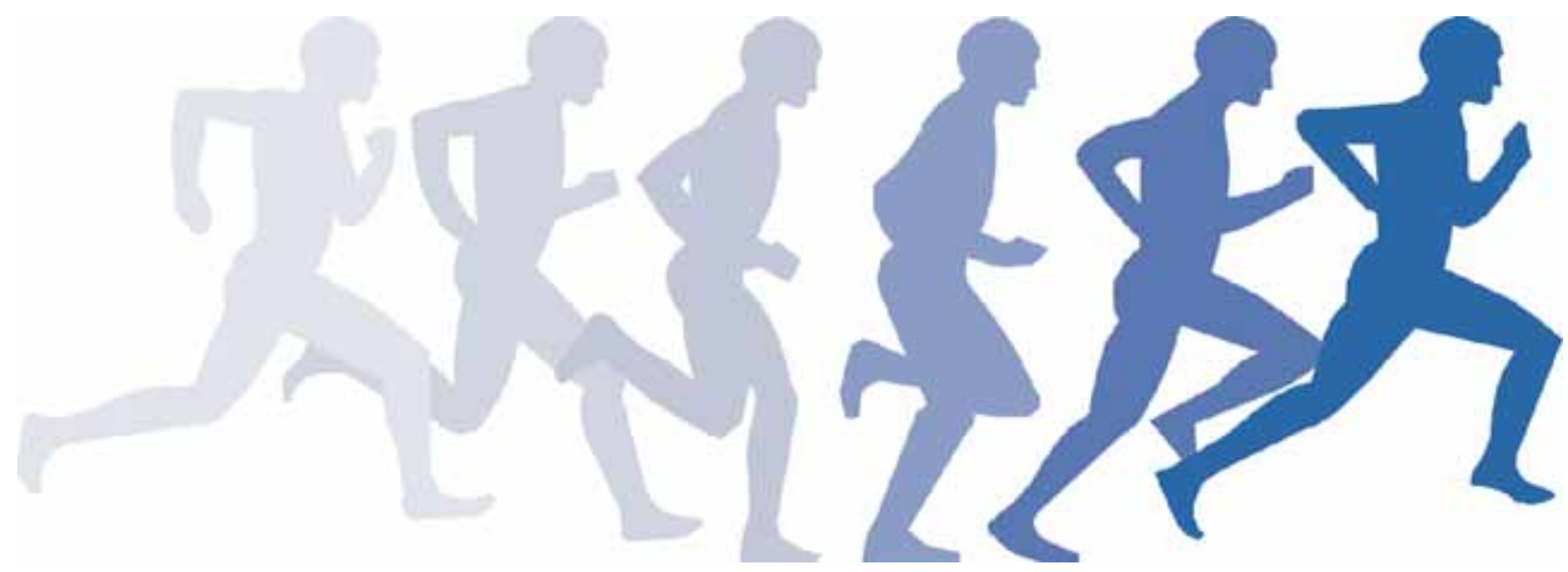